\title{
Total energy, equation of states and bulk modulus of Si and Ge
}

\author{
A.R. Jivani, P.N. Gajjar and A.R. Jani \\ Department of Physics, Sardar Patel University, Vallabh Vidyanagar-388 120, Gujarat, India
}

\begin{abstract}
A model potential describing electron-ion interaction is presented for intrinsic semiconductors $\mathrm{Si}$ and $\mathrm{Ge}$. The present model potential is single parametric, continuous in $\mathbf{r}$-space and weaker within core and Coulombic outside the core. The parameter of the potential is determined using the equilibrium condition at zero pressure. The total energy, equation of states and bulk modulus of Si and Ge are calculated using higher order perturbation theory based on pseudopotential formalism which includes covalent correction term. Numerical results of total energy and bulk modulus obtained for the $\mathrm{Si}$ and $\mathrm{Ge}$ are in good agreements with experimental data and found superior than other such theoretical findings. The predicted equation of states of $\mathrm{Si}$ and $\mathrm{Ge}$ are also excellent.
\end{abstract}

Keywords: pseudopotential method, semiconductors, binding energy, equation of states, bulk modulus

Paper received 08.04.02; accepted for publication 10.12.02.

\section{Introduction}

The second order perturbation theory based on the pseudopotential formalism has been applied to simple metals and their binary alloys, glasses and compounds with many successful examples [1-5]. However, the second order perturbation theory failed for covalent crystals because of covalent bonding character of these crystals. These difficulties were removed with the extension of the perturbation theory in terms of the pseudopotential by taking into account higher order terms corresponding to the covalent bonding effect [6-8]. The treatment of covalent crystals is difficult not because of complex structure of these crystals but mainly due to the determination of the model potential. Soma [9] used this higher order perturbation theory to calculate total energy and bulk modulus of IV semiconductors employing Ashcroft [10] empty core model potential. In addition to the usual potential parameter $R_{c}$, an adjustable additional parameter $R_{c}{ }_{c}$ was used by Soma to ensure minimum energy condition in the first order perturbation energy [9]. He has also reported the results using the Heine-Abarenkov [11] model potential. In all such reported applications $[9,12]$, people have worked with historical model potentials like the Ashcroft and Heine-Abarenkov model potentials. Here, in the present paper, we extended the applications of our single parametric model potential [13] to investigate total energy, equation of states and bulk modulus of $\mathrm{Si}$ and $\mathrm{Ge}$ intrinsic semiconductors using the extended perturbation theory. [13] is

The analytical form of our model potential in $r$-space

$$
W(r)= \begin{cases}-\frac{Z e^{2}}{R_{c}}\left[\frac{3 r}{2 R_{c}}-\frac{r^{2}}{2 R_{c}^{2}}\right] & r<R_{c} \\ -\frac{Z e^{2}}{r} & r \geq R_{c},\end{cases}
$$

where $Z$ is valency of the ion, e is the electronic charge and $R_{c}$ the model potential parameter. The potential parameter $R_{c}$ is determined by using zero pressure condition. The momentum-space representation of this model potential is given by

$W_{b}(\mathrm{q})=-\frac{12 \pi Z e^{2}}{\Omega \mathrm{q}^{3} R_{c}}\left[\frac{\sin \left(\mathrm{q} R_{c}\right)}{2}-\frac{1}{\mathrm{q} R_{c}}+\frac{\sin \left(\mathrm{q} R_{c}\right)}{\left(\mathrm{q} R_{c}\right)^{2}}\right]$.

Where $\Omega$ is the atomic volume and $\mathrm{q}$ is the wave vector.

\section{Computational method}

For a covalent crystal with the diamond structure, based on the higher order perturbation theory, the total energy per electron of the crystal $[6,8]$ is given by

$E=E_{i}+E_{0}+E_{1}+E_{2}+E_{\mathrm{cov}}$

In equation (3), $E_{i}$ is electrostatic energy of point ions immersed in the uniform gas of valence electrons, called the Madelung energy, which is given by 


\section{A.R. Jivani et al.: Total energy, equation of states and bulk modulus od Si and Ge}

$E_{i}=-\frac{\alpha Z^{\frac{5}{3}}}{r_{s}}$,

where $r_{\mathrm{s}}$ is the average interelectronic distance. The Ewald constant $(\alpha)$ for covalent crystals is 1.67085 .

In equation (3), $E_{0}$ is the sum of the kinetic, exchange and correlation energies of the valence electron and is given by

$E_{0}=Z\left[\frac{2.21}{r_{s}^{2}}-\frac{0.916}{r_{s}}-\left(0.115-0.031 \ln \left(r_{s}\right)\right)\right]$

$E_{1}$ is the first order perturbation energy of the valence electron due to the pseudopotential and is given by

$E_{1}=\lim _{\mathrm{q} \rightarrow 0}\left[\frac{4 \pi Z^{2} e^{2}}{\Omega \mathrm{q}^{2}}+Z W_{b}(\mathrm{q})\right]$

For present pseudopotential, the value of the electron-ion interaction is

$E_{1}=\frac{9 \pi Z^{2} e^{2} R_{c}^{2}}{10 \Omega}$

The second order term $E_{2}$ in equation (3) can be written as

$E_{2}=\sum_{\mathrm{q}}\left|W_{s}(\mathrm{q}) S(\mathrm{q})\right| \chi(\mathrm{q}) \varepsilon(\mathrm{q})$,

were the summation $\Sigma^{\prime}$ excludes $\mathrm{q}=0$ and is carried out for 2006 reciprocal vectors. In above equation, structure factor of the diamond lattice is given by

$$
S(\mathrm{q})=\sum_{\mathrm{q}} \delta_{\mathrm{q}, \mathrm{Q}} \cos (\mathrm{q} \cdot \tau)
$$

where $\mathrm{Q}$ is the reciprocal vector and $\tau=(a / 4, a / 4, a / 4)$ is a half of the vector in the body diagonal direction separating the two interpenetrating FCC lattices of lattice constant ' $a$ '.

In equation (8), the pseudopotential screened by the electron gas is

$W_{S}(\mathrm{q})=\frac{W_{b}(\mathrm{q})}{\varepsilon(\mathrm{q})}$

The screening factor $\varepsilon(q)$ for a free electron gas is

$\varepsilon(\mathrm{q})=1-\frac{8 \pi e^{2}}{\Omega \mathrm{q}^{2}}[1-f(\mathrm{q})] \chi(\mathrm{q})$

here $f(q)$ is the local-field correction and $\chi(q)$ is perturbation characteristics which is given by

$\chi(\mathrm{q})=-\frac{3 Z}{4 k_{F}^{2}}\left[0.5+\frac{1-X^{2}}{2 X} \cdot \ln \left|\frac{1+X}{1-X}\right|\right]$,

where $X=\frac{\mathrm{q}}{2 k_{F}}$ and $k_{F}$ is the Fermi wave vector.
The covalent bond correction $\left(E_{\mathrm{cov}}\right)$ corresponding to the third and fourth-order terms to the total energy is given by

$E_{\mathrm{cov}}=-n\left(E_{F}\right) \cdot\left[\left(\frac{a^{2}}{4 \pi^{2}}\right)^{2} W_{s}(111)^{4}+\right.$

$\left.+\left(\frac{a^{2}}{2 \pi^{2}}\right) W_{s}(220) W_{s}(111)^{2}\right]$,

where the valence electron density of states per atom at the Fermi surface $n\left(E_{F}\right)=Z\left(2 E_{F} / 3\right)^{-1}$. In equation (13), $W_{S}(111)$ and $W_{S}(220)$ are the screened pseudopotential Fourier components at reciprocal points (111) and (220). from

The equation of states and bulk modulus are obtained

$P=-\frac{d E}{d \Omega}$

and

$B=\Omega \frac{d^{2} E}{d \Omega^{2}}$

In the present calculations, to determine $R_{c}$, the pressure less than $10^{-3} \mathrm{kbar}$ is taken as equal to zero. For the incorporation of exchange and correlation effects to the dielectric function $\varepsilon(q)$, we adopted seven approximations, proposed by Hartree $(\mathrm{H})$ [14], Hubbard and Sham (HB) [15,16], modified Hubbard Sham (HS) [15-17], Kleinman (K) [18], Kleinman and Langreth (KL) [18, 19], Vashistha and Singwi (VS) [20] and Taylor (T) [21].

\section{Results and discussion}

Tables 1 and 2 show the total energy per electron of semiconductors obtained by using equations (3-13) alongwith experimental and other such reported values [9, 12]. It is found from these tables that the effect of exchange and correlation is clearly distinguishable. The inclusion of exchange and correlation function suppresses the value of total energy of Si and Ge than those obtained by the static $\mathrm{H}$ dielectric function (without exchange and correlation). Thus, decrease in total energy due to inclusion of the exchange correlation to the dielectric function favors the cohesion of semiconductors. It is also predicted that the Taylor's $f(\mathrm{q})$ gives minimum energy for both Si and Ge.

Figures 1 and 2 show the pressure-volume relations for $\mathrm{Si}$ and $\mathrm{Ge}$ alongwith the experimental data [12]. The present finding of equation of states with various $f(\mathrm{q})$ is for $\mathrm{Si}$ and $\mathrm{Ge}$ at different volume are within the value obtained by inclusion of $\mathrm{H}$ and $\mathrm{T}$ local field correction to the dielectric function. Hence, to avoid the complication in the figures, equation of states obtained due to $\mathrm{H}$ and $\mathrm{T}$ are plotted. At $9 \%$ compression the effect of exchange and correlation function of $\mathrm{T}$ with respect to $\mathrm{H}$ is $25 \%$ for $\mathrm{Si}$ and $28 \%$ for $\mathrm{Ge}$. Our equation of states for $\mathrm{Si}$ and 


\section{A.R. Jivani et al.: Total energy, equation of states and bulk modulus od Si and Ge}

Table 1. Total energy $E$ per electron of Si (in Rydberg units)

\begin{tabular}{|c|c|c|c|c|c|}
\hline \multirow{2}{*}{$f(q)$} & \multirow{2}{*}{$\begin{array}{c}\text { Present } \\
\text { [9] }\end{array}$} & \multicolumn{2}{|c|}{ Others } & \multirow[b]{2}{*}{ [9] } & \multirow[t]{2}{*}{ Observed } \\
\hline & & [9] & [12] & & \\
\hline$\overline{\mathrm{H}}$ & -1.9413 & - & - & -1.9758 & \multirow{7}{*}{-1.980} \\
\hline $\mathrm{HB}$ & -2.0458 & -1.9758 & -1.9882 & - & \\
\hline HS & -2.0440 & -1.9734 & -1.9859 & - & \\
\hline $\mathrm{K}$ & -2.0898 & -1.9965 & -2.0078 & - & \\
\hline $\mathrm{KL}$ & -2.0890 & -1.9965 & -2.0041 & - & \\
\hline$\underline{\mathrm{VS}}$ & -2.1237 & -2.0003 & -2.0105 & - & \\
\hline $\mathrm{T}$ & -2.1463 & - & - & - & \\
\hline
\end{tabular}

Ge are compared with the experimental data reported by Bridgman, Vaidya et al (by piston-cylinder method) and Senoo (by X-ray diffraction method)[12]. The excellent agreement for equation of states of $\mathrm{Si}$ and $\mathrm{Ge}$ is concluded in the present study.

The presently investigated bulk modulus (B) of semiconductors is tabulated in Tables 3 and 4 alongwith other such theoretical and observed data $[6,9]$. The values in the parenthesis show the percentile deviation from the experimental data. From tables 3 and 4, it is noticed that the deviation of our result from observed values is $1.64 \%$ to $18.25 \%$ for $\mathrm{Si}$ and $2.14 \%$ to $33.79 \%$ for Ge. The results due to others $[6,9]$ include the deviation ranging from $14.72 \%$ to $31.59 \%$ for Si and $0.94 \%$ to $25.73 \%$ for Ge from the observed values. From Tables 1 to 4 , we conclude that though the calculated values of total energy and bulk modulus of $\mathrm{Si}$ and $\mathrm{Ge}$ scatter in a bit wide range around the observed values, present findings are more improved results as com-
Table 2. Total energy $E$ per electron of Ge (in Rydberg units)

\begin{tabular}{|c|c|c|c|c|c|}
\hline$f(\mathrm{q})$ & Present & & Others & & Observed \\
\hline & [9] & [9] & [12] & [9] & \\
\hline$\overline{\mathrm{H}}$ & -1.8617 & - & - & -1.960 & \\
\hline $\mathrm{HB}$ & -1.9608 & -1.9418 & -1.9663 & - & \\
\hline HS & -1.9605 & -1.9404 & -1.9647 & - & \\
\hline $\mathrm{K}$ & -2.0010 & -1.9654 & -1.9874 & - & -1.978 \\
\hline $\mathrm{KL}$ & -2.0037 & -1.9635 & -1.9852 & - & \\
\hline$\underline{\mathrm{VS}}$ & -2.0397 & -1.9736 & -1.9933 & - & \\
\hline $\mathrm{T}$ & -2.0597 & - & - & - & \\
\hline
\end{tabular}

pared to previously reported other such theoretical values $[6,9,12]$. Thus, the present study confirms the fruitfulness of our model potential in predicting electronic properties of semiconductors. This also provides better set of theoretical results on total energy, equation of states and bulk modulus for intrinsic semiconductors $\mathrm{Si}$ and $\mathrm{Ge}$ for further comparison either with theoretical or experimental results.

\section{Acknowledgements}

The work is supported under the special assistance programme at the level of Departmental research support by the University Grants Commission, New Delhi, INDIA. The author (PNG) acknowledges the facilities availed at the Abdul Salam International center for Theoretical Physics, ITALY, during the course of the work.

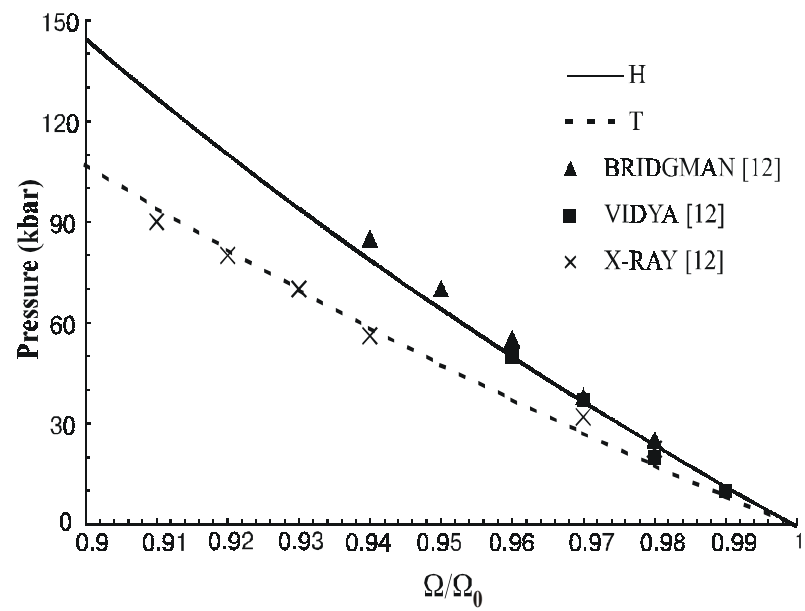

Fig. 1. The pressure-volume relations of $\mathrm{Si}$

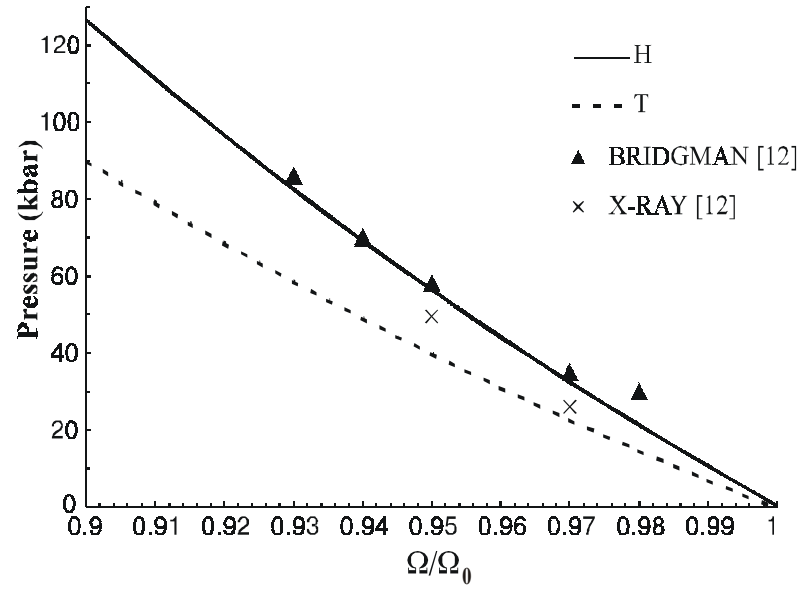

Fig. 2. The pressure-volume relations of $\mathrm{Ge}$ 
A.R. Jivani et al.: Total energy, equation of states and bulk modulus od Si and Ge

Table 3. The bulk modulus of Si (in $10^{12} \mathrm{dyne} / \mathrm{cm}^{2}$ )

\begin{tabular}{|c|c|c|c|c|c|}
\hline$f(\mathrm{q})$ & Present & [6] & $\begin{array}{c}\text { Others } \\
{[9]}\end{array}$ & [9] & $\begin{array}{c}\text { Observed } \\
{[9]}\end{array}$ \\
\hline $\mathrm{H}$ & $1.1565(18.25)$ & $\begin{array}{l}0.833 \\
(14.83)\end{array}$ & - & - & \multirow{7}{*}{0.978} \\
\hline $\mathrm{HB}$ & 1.0315 & $\begin{array}{l}0.775 \\
(20.76)\end{array}$ & $\begin{array}{l}0.852 \\
(12.88)\end{array}$ & $\begin{array}{l}0.801 \\
(18.1)\end{array}$ & \\
\hline HS & $1.0041(2.67)$ & $\begin{array}{l}0.729 \\
(25.46)\end{array}$ & $\begin{array}{l}0.834 \\
(14.72)\end{array}$ & $\begin{array}{l}0.780 \\
(20.24)\end{array}$ & \\
\hline $\mathrm{K}$ & $0.9940(1.64)$ & - & $\begin{array}{l}0.810 \\
(17.18)\end{array}$ & $\begin{array}{l}0.754 \\
(22.90)\end{array}$ & \\
\hline KL & $0.9369(4.20)$ & $\begin{array}{l}- \\
(20.86)\end{array}$ & $\begin{array}{l}0.774 \\
(26.69)\end{array}$ & 0.717 & \\
\hline VS & $0.9176(6.17)$ & $\begin{array}{l}- \\
(26.07)\end{array}$ & $\begin{array}{l}0.723 \\
(31.59)\end{array}$ & 0.669 & \\
\hline $\mathrm{T}$ & $0.8625(11.81)$ & - & - & - & \\
\hline
\end{tabular}

\section{References}

1. P. N. Gajjar, B. Y. Thakore and A. R. Jani, Total crystal energy and heat of solution of alkali based binary alloys // Acta Phys. Polo. A, 99 (5), pp. 565 - 578 (2001).

2. Minal. H. Patel, A. M. Vora, P. N. Gajjar and A. R. Jani, Fermi energy and Fermi surface distortion of the Cs-K, Cs-Rb and RbK binary systems // Physica B, 304, pp. 152 - 158 (2001).

3. B. Y. Thakore, P. N. Gajjar and A. R. Jani, Collective modes in $\mathrm{Ca}_{70} \mathrm{Mg}_{30}$ glass // Bull. Mater. Sci., 23 (1), pp. 5 - 9 (2000).

4. P. N. Gajjar, B. Y. Thakore and A. R. Jani, Total crystal energy of some simple metals // Rom. J. Phys., 43 (9-10), pp. $747-$ 753 (1998)

5. V. T. Shvets and E. V. Belov, Choice of pseudopotential and electroresistance of simple disordered metals // Acta Phys. Polo. A, 96, pp. 741-750 (1999)

6. Akita Morita, T. Soma and T. Takeda, Perturbation theory of covalent crystals: I. Calculation of cohesive energy and compressibility // J. of Phys. Soc. of Japan, 32 (1), pp. 29 - 37 (1972).

7. G. P. Srivastava, The Physics of Phonons, IOP Publishing Ltd, Bristol (1990).

8. T. Soma, The electronic theory of III-V and II-VI tetrahedral compounds: I. Crystal energy and bulk modulus // J. Phys. C, 11 (13), pp. 2669-2678 (1978)

9. T. Soma, Local Heine - Abarenkov Model potential for covalent crystals // Phys. Stat. Sol. (b), 86, pp. 263 - 268 (1978).

10. N. W. Ashcroft, Electron - ion pseudopotentials in metals // Phys. Lett., 23 (1), pp. 48-50 (1966).

11. V. Heine and I. V. Abarenkov, A new method for the electronic structure of metals // Phil. Mag., 9, pp. 451-465 (1964).
Table 4. The bulk modulus of Ge (in $10^{12} \mathrm{dyne} / \mathrm{cm}^{2}$ )

\begin{tabular}{|c|c|c|c|c|c|}
\hline \multirow[t]{2}{*}{$f(\mathrm{q})$} & \multirow[t]{2}{*}{ Present } & \multicolumn{3}{|c|}{ Others } & \multirow{2}{*}{$\begin{array}{c}\text { Observed } \\
\text { [9] }\end{array}$} \\
\hline & & [6] & [9] & [9] & \\
\hline $\mathrm{H}$ & 1.0035 (33.79) & $\begin{array}{l}0.757 \\
(0.94)\end{array}$ & - & - & \multirow{7}{*}{0.750} \\
\hline HB & $0.8810(17.17)$ & $\begin{array}{l}0.680 \\
(9.33)\end{array}$ & $\begin{array}{l}0.728 \\
(2.93)\end{array}$ & $\begin{array}{l}0.685 \\
(8.67)\end{array}$ & \\
\hline HS & $0.8554(14.06)$ & $\begin{array}{l}0.654 \\
(12.8)\end{array}$ & $\begin{array}{l}0.717 \\
(4.40)\end{array}$ & $\begin{array}{c}0.662 \\
(11.73)\end{array}$ & \\
\hline K & 0.8455 (12.74) & - & $\begin{array}{r}0.704 \\
(6.13)\end{array}$ & $\begin{array}{l}0.646 \\
(13.87)\end{array}$ & \\
\hline $\mathrm{KL}$ & 0.7924 ( 5.65) & - & $\begin{array}{c}0.661 \\
(11.87)\end{array}$ & $\begin{array}{c}0.603 \\
(19.60)\end{array}$ & \\
\hline VS & $0.7661(2.14)$ & - & $\begin{array}{c}0.615 \\
(18.00)\end{array}$ & $\begin{array}{l}0.557 \\
(25.73)\end{array}$ & \\
\hline $\mathrm{T}$ & $0.7218(3.76)$ & - & - & - & \\
\hline
\end{tabular}

12. Masafumi Senoo, Hisao Mii and Ikuya Fujishiro, Calculations of pressure-volume relations for some cubic metals in pseudopotential method // J. of Phys. Soc. of Japan, 41 (5), pp. 1562-1569 (1976).

13. V. N. Patel, Minal H. Patel, B. Y. Thakore, P. N. Gajjar and A. R. Jani, On the application of pseudo-alloy-atom model to the lithium based binary alkali alloys // Solid State Physics (India), 42 C, pp. 283-284 (1999).

14. W. A. Harrison, Elementary electronic structure, World scientific, Singapore (1998).

15. J. Hubbard, The description of the collective motions in terms of many - body perturbation theory II. The correlation energy of a free - electron gas // Proc. Roy. Soc., London A, 243, pp. 336-352 (1958).

16. L. J. Sham, A calculation of the phonon frequencies in Sodium // Proc. Roy. Soc., London, A, 283 (1392), pp. 33 - 49 (1965).

17. D.J.W. Geldart and S.H. Vosko, The screening function of an interaction electron gas // Can. J. Phys., 44 (9), pp. 2137 2171 (1966).

18. L. Kleinman, New approximation for screened exchange and the dielectric constants of metals // Phys. Rev., 160 (3), pp. 585-590 (1967).

19. D. Langreth, Approximate screening function in metals // Phys. Rev., 181 (2), pp. 753-762 (1969).

20. P. Vashishta and K. S. Singwi, Electron correlations at metallic densities // Phys. Rev. B, 6 (3), pp. 875-887 (1972).

21. R. Taylor, A simple useful analytical form of the static electron gas dielectric function // J. Phys. F, 8, pp. 1699-1702 (1978). 